Journal for

.... ImmunoTherapy of Cancer

\title{
Avelumab as second-line therapy for metastatic, platinum-treated urothelial carcinoma in the phase Ib JAVELIN Solid Tumor study: 2-year updated efficacy and safety analysis
}

Andrea B Apolo (D) , ${ }^{1}$ John A Ellerton, ${ }^{2}$ Jeffrey R Infante, ${ }^{3}$ Manish Agrawal, ${ }^{4}$ Michael S Gordon, ${ }^{5}$ Raid Aljumaily, ${ }^{3,6}$ Theodore Gourdin, ${ }^{7}$ Luc Dirix, Keun-Wook Lee, ${ }^{9}$ Matthew H Taylor, ${ }^{10}$ Patrick Schöffski, ${ }^{11,12}$ Ding Wang, ${ }^{13}$ Alain Ravaud (1) , ${ }^{14}$ Juliane Manitz, ${ }^{15}$ Gregory Pennock, ${ }^{16}$ Mary Ruisi, ${ }^{15}$ James L Gulley (D) , ${ }^{1}$ Manish R Patel ${ }^{17}$

To cite: Apolo AB, Ellerton JA, Infante JR, et al. Avelumab as second-line therapy for metastatic, platinum-treated urothelial carcinoma in the phase Ib JAVELIN Solid Tumor study: 2-year updated efficacy and safety analysis. Journal for ImmunoTherapy of Cancer 2020;8:e001246. doi:10.1136/ jitc-2020-001246

- Additional material is published online only. To view, please visit the journal online (http://dx.doi.org/10.1136/jitc2020-001246).

Accepted 18 August 2020

Check for updates

(c) Author(s) (or their employer(s)) 2020. Re-use permitted under CC BY-NC. No commercial re-use. See rights and permissions. Published by BMJ.

For numbered affiliations see end of article.

Correspondence to

Dr Andrea B Apolo;

andrea.apolo@nih.gov

\section{ABSTRACT}

Background Anti-programmed cell death ligand 1 (PDL1)/programmed cell death 1 antibodies have shown clinical activity in platinum-treated metastatic urothelial carcinoma, resulting in regulatory approval of several agents, including avelumab (anti-PD-L1). We report $\geq 2$-year follow-up data for avelumab treatment and exploratory subgroup analyses in patients with urothelial carcinoma.

Methods Patients with previously treated advanced/ metastatic urothelial carcinoma, pooled from two cohorts of the phase Ib JAVELIN Solid Tumor trial, received avelumab $10 \mathrm{mg} / \mathrm{kg}$ every 2 weeks until disease progression, unacceptable toxicity or withdrawal. End points included best overall response and progression-free survival (PFS) per RECIST V.1.1, overall survival (OS) and safety. Post hoc analyses included objective response rates (ORRs) in subgroups defined by established high-risk/poorprognosis characteristics and association between time to response and outcome.

Results 249 patients received avelumab; efficacy was assessed in 242 postplatinum patients. Median follow-up was 31.9 months (range 24-43), and median treatment duration was 2.8 months (range $0.5-42.8$ ). The confirmed ORR was $16.5 \%$ (95\% Cl $12.1 \%$ to $21.8 \%$; complete response in $4.1 \%$ and partial response in $12.4 \%$ ). Median duration of response was 20.5 months $(95 \% \mathrm{Cl} 9.7$ months to not estimable). Median PFS was 1.6 months $(95 \% \mathrm{Cl} 1.4$ to 2.7 months) and the 12-month PFS rate was $16.8 \%$ (95\% Cl $11.9 \%$ to $22.4 \%$ ). Median OS was 7.0 months ( $95 \% \mathrm{Cl} 5.9$ to 8.5 months) and the 24-month OS rate was $20.1 \%$ (95\% Cl $15.2 \%$ to $25.4 \%)$. In post hoc exploratory analyses, avelumab showed antitumor activity in high-risk subgroups, including elderly patients and those with renal insufficiency or upper tract disease; ORRs were numerically lower in patients with liver metastases or low albumin levels. Objective response achieved by 3 months versus later was associated with longer OS (median not reached $(95 \% \mathrm{Cl} 18.9$ months to not estimable) vs 7.1 months ( $95 \% \mathrm{Cl} 5.2$ to 9.0 months)).
Safety findings were consistent with previously reported 6-month analyses.

Conclusions After $\geq 2$ years of follow-up, avelumab showed prolonged efficacy and acceptable safety in patients with platinum-treated advanced/metastatic urothelial carcinoma, including high-risk subgroups. Survival appeared longer in patients who responded within 3 months. Long-term safety findings were consistent with earlier reports with avelumab treatment in this patient population.

\section{INTRODUCTION}

Patients with advanced urothelial cancer (UC) who have disease progression after platinum-based chemotherapy have a poor prognosis. Established factors that predict shorter overall survival (OS) include Eastern Cooperative Oncology Group performance status (ECOG PS) $\geq 1$, hemoglobin level $<100 \mathrm{~g} / \mathrm{L}$ and presence of liver metastasis; these factors are used to determine the Bellmunt risk score. ${ }^{1}$ Other factors associated with high-risk disease or poor response to chemotherapy include upper tract disease, ${ }^{2}$ renal insufficiency, ${ }^{3}$ older age ${ }^{4}$ and albumin level. ${ }^{56}$

Antibodies that bind to programmed cell death protein 1 (PD-1) or its ligand (PD-L1) are clinically active treatments for patients with advanced UC. ${ }^{7-14}$ Avelumab, an antiPD-L1 antibody, has shown antitumor activity and acceptable safety in a range of advanced solid tumors. ${ }^{9} 10^{15-18}$ In a subgroup of 161 patients with platinum-treated, advanced/ metastatic UC enrolled in a large phase I trial (JAVELIN Solid Tumor), the objective response rate (ORR) after $\geq 6$ months of 
follow-up was $17 \%$ and responses occurred across various subgroups. ${ }^{10}$ These data led to the accelerated approval of avelumab by the US Food and Drug Administration in this setting. ${ }^{19}$

In studies of checkpoint inhibitors, the identification of potential surrogate end points for OS has been a topic of high interest across multiple tumor types. Systematic reviews and meta-analyses have concluded that, although responders to checkpoint inhibitors tend to have longer OS than non-responders, including patients with UC, the correlation was weak. ${ }^{20-22}$ However, few studies have assessed whether early response is associated with longer OS in UC. A retrospective analysis of first-line chemotherapy for UC concluded that early response may not predict OS. ${ }^{23}$ However, in studies of second-line avelumab in patients with other cancers (metastatic Merkel cell carcinoma or unresectable mesothelioma), objective response achieved within 2-3 months was associated with longer OS compared with patients without an early response. ${ }^{24} 25$

Here, we report an updated analysis of avelumab in patients with previously treated UC with a minimum of 24 months of follow-up. We also report exploratory post hoc analyses evaluating high-risk subgroups and the association between early response and long-term outcomes.

\section{METHODS}

\section{Study design and procedures}

JAVELIN Solid Tumor (NCT01772004) was a phase I, open-label, multicohort trial assessing avelumab monotherapy for various solid tumors. The study design and full methodology in UC cohorts have been reported previously. ${ }^{10}$ Briefly, patients with locally advanced or metastatic urothelial carcinoma, disease progression following platinum-based chemotherapy or platinum ineligible, and no prior immunotherapy, received avelumab $10 \mathrm{mg}$ / $\mathrm{kg}$ by 1 hour intravenous infusion every 2 weeks until confirmed disease progression, unacceptable toxicity or other protocol-specified criteria for withdrawal occurred. Premedication with an antihistamine (diphenhydramine or equivalent) and acetaminophen was administered 30-60 min prior to each avelumab infusion. Dose modifications of avelumab (reductions or increases) were not permitted. Tumor response and progression were assessed per Response Evaluation Criteria in Solid Tumors V.1.1 by a blinded independent review committee. In exploratory post hoc analyses, clinical activity was assessed in subgroups defined by standard baseline characteristics or characteristics associated with high-risk disease or poor response to chemotherapy. PD-L1 expression in tumor samples was assessed using a research-only assay (Dako PD-L1 IHC 73-10 pharmDx), and PD-L1+ status was defined prospectively as expression on $\geq 5 \%$ of tumor cells, consistent with previous analyses. ${ }^{9}{ }^{10}$ Adverse events (AEs) were graded according to National Cancer Institute Common Terminology Criteria for Adverse Events V.4.0. Immune-related AEs (irAEs) were identified using a prespecified list of Medical Dictionary for Regulatory Activities preferred terms, followed by comprehensive medical review using predefined criteria. Infusion-related reactions (IRRs) and related events were identified via an expanded definition that included prespecified Medical Dictionary for Regulatory Activities terms (IRR, drug hypersensitivity, anaphylactic reaction, hypersensitivity and type 1 hypersensitivity) occurring on the day of or the day after the infusion, in addition to signs and symptoms of IRR that occurred on the day of infusion and resolved within 2 days.

The primary end point for this study was the occurrence of dose-limiting toxicities during the first 3 weeks of avelumab treatment in the dose-escalation part of the study (reported previously), ${ }^{26}$ and best overall response per RECIST V.1.1, adjudicated by independent end point review committee, in efficacy expansion cohorts. Secondary end points included duration of response (DOR), progression-free survival (PFS), OS, tumor PD-L1 expression and safety.

\section{Statistical analysis}

Time-to-event end points (DOR, PFS and OS) were analyzed using the Kaplan-Meier method. For post hoc subgroup analyses, ORR (proportion of patients with a complete response (CR) or partial response (PR)) and disease control rate (DCR; proportion of patients with CR, $\mathrm{PR}$, stable disease (SD) or non-CR/non-progressive disease (PD) ) were analyzed using event proportions, and DCR and PFS were assessed as sensitivity analyses. Additionally, a sensitivity analysis was performed for post hoc subgroups based on continuous covariates, including creatinine clearance and albumin concentration; an automated cutoff search was applied using recursive partitioning to test the robustness of cut-offs selected. ${ }^{27}$ This data-mining approach conditionally partitions the covariate space that best predicts the end point of interest. DCR was selected to avoid redundancy and use classification tree models. The partition algorithm selected the input variable that had the strongest association with disease control and implemented a binary split in the selected input variable; this process was repeated recursively. Post hoc analysis of the association between time to response (TTR) and DOR or OS was performed using landmark Kaplan-Meier analysis. Associations were assessed visually using a scatter plot matrix for TTR, DOR, PFS and OS (data not shown). Subgroups were defined based on whether response occurred by week 7 (first tumor assessment \pm 5 days; DOR analysis) or by 3 months (12 weeks; second tumor assessment \pm 5 days; OS analysis), and analyses included only those patients who remained on study at the defined time point. Univariate and multivariate Cox regression model analyses were also performed (data not shown). Following methodology based on previously published studies, further OS analyses were carried out that defined separate categories for patients with early response versus $\mathrm{SD} /$ late response versus other patients, and for patients who continued treatment beyond PD versus patients who 
discontinued treatment when PD occurred..$^{28}$ OS analyses were confirmed by supplementary analyses, including one using an alternative landmark definition (week 7 , as above) and another that excluded patients who were not evaluable by blinded IRC.

\section{RESULTS}

\section{Patient characteristics and disposition}

Between September 3, 2014, and March 15, 2016, 329 patients with advanced/metastatic UC were screened for enrollment into two study cohorts; of those, 249 met eligibility criteria and received avelumab (table 1 ; online additional file 1). Detailed patient characteristics for the pooled population have been reported previously. ${ }^{10}$ Briefly, median age was 68 years (range 30-89 years), most patients were male $(178(71.5 \%))$, and $124(49.8 \%)$ had received $\geq 2$ prior lines of therapy for advanced disease. At data cut-off on April 10, 2018, median follow-up was 31.9 months (range 24-43 months). Patients received a median of six doses of avelumab (range 1-93 doses) for a median of 2.8 months (range $0.5-42.8$ months). Twelve patients $(4.8 \%)$ remained on treatment at last follow-up. The most common reason for treatment discontinuation was disease progression (155 (62.2\%)); other reasons were AEs $(39(15.7 \%))$, death (13 (5.2\%)), withdrawal of consent (13 $(5.2 \%))$, protocol non-compliance (2 $(0.8 \%))$ and other reasons $(15(6.0 \%))$. Consistent with the approved label of avelumab and a previous report, ${ }^{10}$ seven platinum-naïve patients were excluded from efficacy analyses but were included in safety analyses.

\section{Antitumor activity}

Of 242 postplatinum patients, 40 had a confirmed response (CR in $10(4.1 \%)$; PR in $30(12.4 \%)$ ), resulting in an ORR of $16.5 \%$ (95\% CI $12.1 \%$ to $21.8 \%$; online additional file 2). Data for best overall response were missing or not assessable in 43 patients $(17.8 \%$; no postbaseline tumor assessment in 35, postbaseline assessment but response not evaluable in 2, stable disease of insufficient duration in 5 and non-evaluable with PD $>12$ weeks after study assessment in 1). Median TTR was 2.7 months (range 1.3-11.0 months). At last follow-up, response was ongoing or considered SD in 20 of 40 responding patients (50.0\%; online additional file 3A). Median DOR was 20.5 months (95\% CI 9.7 months to not estimable), and the estimated proportion of responses lasting $\geq 12$ months was $65.4 \%$ (95\% CI $47.0 \%$ to $78.8 \%$ ). Of 198 evaluable patients with a baseline and on-study tumor assessment, $54(27.3 \%)$ had tumor shrinkage of $\geq 30 \%$ (online additional file 3B). In patients with $\leq 1 \quad(n=118), 2(n=73)$ or $\geq 3 \quad(n=51)$ previous lines of therapy for advanced disease, ORRs (95\% CIs) were $19.5 \%$ ( $12.8 \%$ to $27.8 \%), 16.4 \%$ $(8.8 \% \%$ to $27.0 \%)$ and $9.8 \%$ (3.3\% to $21.4 \%)$, respectively. Median PFS was 1.6 months (95\% CI 1.4 to 2.7 months), and PFS rates at 6 and 12 months were $26.5 \%$
Table 1 Patient demographics and baseline characteristics

\begin{tabular}{|c|c|}
\hline Characteristic & $\mathrm{N}=\mathbf{2 4 9}$ \\
\hline Median age (range), years & $69.0(30-89)$ \\
\hline$<75, \mathrm{n}(\%)$ & $176(70.7)$ \\
\hline$\geq 75, \mathrm{n}(\%)$ & $73(29.3)$ \\
\hline \multicolumn{2}{|l|}{ Sex, n (\%) } \\
\hline Male & $178(71.5)$ \\
\hline Female & $71(28.5)$ \\
\hline \multicolumn{2}{|l|}{ ECOG PS, n (\%) } \\
\hline 0 & $88(35.3)$ \\
\hline 1 & $161(64.7)$ \\
\hline Median time since first diagnosis (range), months & $20.4(1.9-289.2)$ \\
\hline $\begin{array}{l}\text { Median time since diagnosis of metastatic disease } \\
\text { (range), months }\end{array}$ & $12.0(0.6-70.7)$ \\
\hline \multicolumn{2}{|l|}{ Prior platinum therapy, $\mathrm{n}(\%)$} \\
\hline Cisplatin & $153(61.4)$ \\
\hline Carboplatin & $122(49.0)$ \\
\hline \multicolumn{2}{|l|}{ No. of prior lines for advanced disease ${ }^{*}$} \\
\hline$\leq 1, \mathrm{n}(\%)$ & $119(47.8)$ \\
\hline $2, n(\%)$ & $72(28.9)$ \\
\hline$\geq 3, \mathrm{n}(\%)$ & $52(20.9)$ \\
\hline Median (range) & $2(0-6)$ \\
\hline \multicolumn{2}{|l|}{ Site of primary tumor, $\mathrm{n}(\%)$} \\
\hline Upper tract (renal pelvis/ureter) & $58(23.3)$ \\
\hline Lower tract (bladder/urethra) & $191(76.7)$ \\
\hline \multicolumn{2}{|l|}{ Visceral metastasis at baseline, $\mathrm{n}(\%) \dagger$} \\
\hline Present & $210(84.3)$ \\
\hline Absent & $39(15.7)$ \\
\hline \multicolumn{2}{|l|}{ Liver metastasis at baseline, $\mathrm{n}(\%)$} \\
\hline Present & $84(33.7)$ \\
\hline Absent & $165(66.3)$ \\
\hline \multicolumn{2}{|l|}{ Renal function, $\mathrm{n}(\%)$} \\
\hline $\mathrm{CrCl} \geq 60 \mathrm{~mL} / \mathrm{min}$ & $132(53.0)$ \\
\hline $\mathrm{CrCl}<60 \mathrm{~mL} / \mathrm{min}$ & $113(45.4)$ \\
\hline Missing & $4(1.6)$ \\
\hline \multicolumn{2}{|l|}{ Albumin level, $\mathrm{n}(\%)$} \\
\hline$<35 \mathrm{~g} / \mathrm{L}$ & $46(18.6)$ \\
\hline$\geq 35 \mathrm{~g} / \mathrm{L}$ & $203(81.5)$ \\
\hline \multicolumn{2}{|l|}{ Bellmunt risk score, $\mathrm{n}(\%)$} \\
\hline 0 & $58(23.3)$ \\
\hline 1 & $114(45.8)$ \\
\hline 2 & $59(23.7)$ \\
\hline 3 & $18(7.2)$ \\
\hline \multicolumn{2}{|l|}{ Tumor PD-L1 expression ( $\geq 5 \%$ cut-off), $n(\%)$} \\
\hline Positive & $85(34.1)$ \\
\hline Negative & $135(54.2)$ \\
\hline Not evaluable & $29(11.6)$ \\
\hline
\end{tabular}

Baseline was defined as $\leq 28$ days prior to the first dose of study treatment. *Excludes platinum-naïve patients.

†Defined as metastasis in any non-lymph node site.

$\mathrm{CrCl}$, creatinine clearance; ECOG PS, Eastern Cooperative Oncology Group performance status; PD-L1, programmed cell death ligand 1.

(95\% CI $20.9 \%$ to $32.5 \%$ ) and $16.8 \%$ (95\% CI $11.9 \%$ to $22.4 \%$ ), respectively (figure $1 \mathrm{~A}$ ). Median OS was 7.0 months (95\% CI 5.9 to 8.5 months), and OS rates at 12 
A
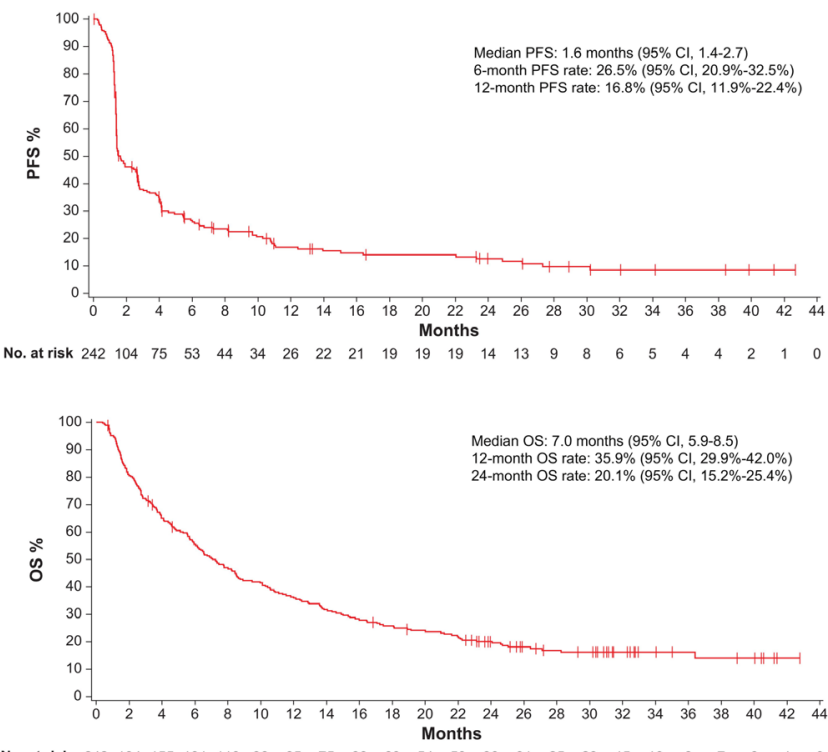

No. at risk $242194 \begin{array}{lllllllllllllllllllll}155 & 131 & 110 & 98 & 85 & 75 & 66 & 60 & 54 & 50 & 39 & 31 & 25 & 23 & 15 & 10 & 8 & 7 & 6 & 1\end{array}$

Figure 1 Survival in postplatinum patients after median duration of follow-up of 31.9 months (range 24-43 months). (A) Progression-free survival (PFS) and (B) overall survival (OS); $n=242$.

and 24 months were $35.9 \%$ (95\% CI $29.9 \%$ to $42.0 \%)$ and $20.1 \%$ (95\% CI $15.2 \%$ to $25.4 \%$ ), respectively (figure $1 \mathrm{~B}$ ).

\section{PD-L1 biomarker analysis}

For PD-L1 expression, 214 patients $(88.4 \%)$ were evaluable, of whom $84(39.3 \%)$ had PD-L1+ tumors and 130 $(60.7 \%)$ had PD-L1 - tumors. Efficacy findings in PD-L1 subgroups are shown in online additional files $3 \mathrm{~B}$ and 4.

\section{High-risk subgroup analyses}

Responses to avelumab occurred in all subgroups defined by individual risk factors (figure 2). Factors associated with a higher ORR compared with respective comparator subgroups were absence of baseline visceral or liver metastases, older age ( $\geq 75$ years) and high albumin $(\geq 35 \mathrm{~g} / \mathrm{L})$. ORRs were similar within subgroups defined by renal function (creatinine clearance $<60$ or $\geq 60 \mathrm{~mL} / \mathrm{min}$ ) and tumor location (upper vs lower tract). In patients with a Bellmunt prognostic (or risk) score of $0,1,2$ or $3{ }^{1}$ ORRs $(95 \% \mathrm{CI})$ were $22.2 \%(12.0 \%$ to $35.6 \%), 21.4 \%$ $(14.2 \% \%$ to $30.2 \%), 6.9 \%(1.9 \%$ to $16.7 \%)$ and $0 \%(0 \%$ to $18.5 \%)$, respectively. DCR and PFS were analyzed in selected subgroups (online additional files 5 and 6). Similar to ORR analyses, no difference in DCR or PFS was found between subgroups defined by renal function or tumor location, and patients without baseline liver metastases had a higher DCR and longer PFS than those with liver metastases. In contrast to ORR analyses, subgroups defined by age or albumin level showed no differences in DCR or PFS. Data-driven cut-off searches yielded no new relevant subgroups, and cut-offs found using recursive partitioning were similar to predefined cut-offs (data not shown).

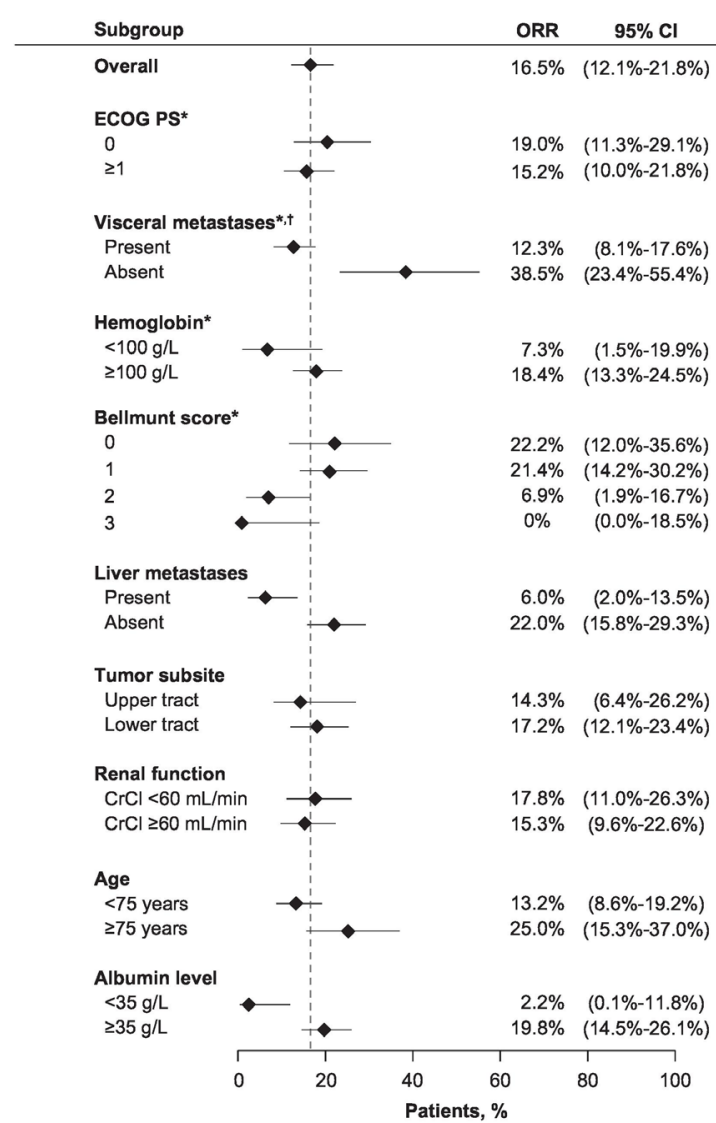

Figure 2 Objective response rates (ORRs) in selected highrisk and poor-prognosis subgroups. *Prespecified subgroups. ${ }^{\dagger}$ Lung, liver or bone metastases.

\section{Association between early response and long-term outcomes}

In a post hoc exploratory analysis comparing DOR in patients who had achieved an objective response (CR or PR) either by week 7 (first tumor assessment; $n=15$ ) or after that time point $(n=25)$, the median DOR was 25.9 months (95\% CI 9.0 months to not estimable) vs 20.5 months (95\% CI 8.1 months to not estimable), respectively. CIs were large due to the small sample size. Post hoc landmark analyses were also performed to assess the association between response by 3 months of treatment (second tumor assessment) and OS. In patients who had an objective response by this time point, the median duration of OS beyond 3 months was not reached (95\% CI 18.9 months to not estimable) vs 7.1 months (95\% CI 5.2 to 9.0 months) in patients who did not respond by 3 months (figure 3). To investigate this observation in more detail, patients without response by 3 months were further categorized according to whether they had SD by 3 months and/or achieved a later response, or whether their best response was PD or not evaluable; median duration of OS beyond 3 months in these subgroups was 9.5 months (95\% CI 6.5 to 14.4 months) and 4.3 months (95\% CI 2.8 to 7.1 months), respectively (figure 3). Further analyses found that OS was similar between patients with PD who continued treatment beyond investigator-confirmed PD and those who did not (figure 3), although this analysis might be biased because the decision to continue 

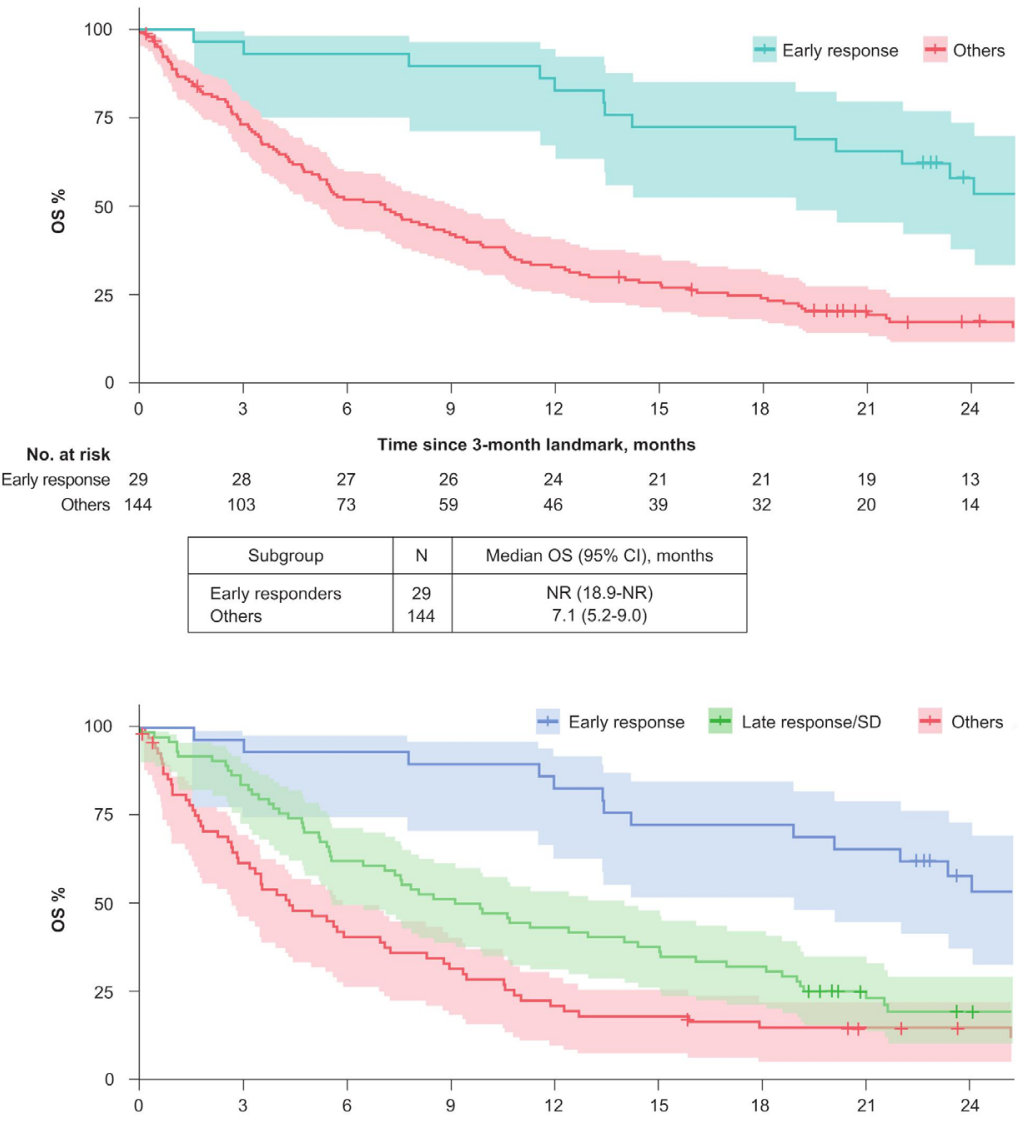

No. at risk

Early response 29 Late response/SD 74

Time since 3-month landmark, months

Others 70

$\begin{array}{lll}28 & 27 & 26\end{array}$

$62-46$

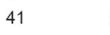

\begin{tabular}{|l|c|c|}
\hline \multicolumn{1}{|c|}{ Subgroup } & $\mathrm{N}$ & Median OS (95\% Cl), months \\
\hline Early responders & 29 & $\mathrm{NR}(18.9-\mathrm{NR})$ \\
Late response/SD & 74 & $9.5(6.5-14.4)$ \\
Others & 70 & $4.3(2.8-7.1)$ \\
\hline
\end{tabular}
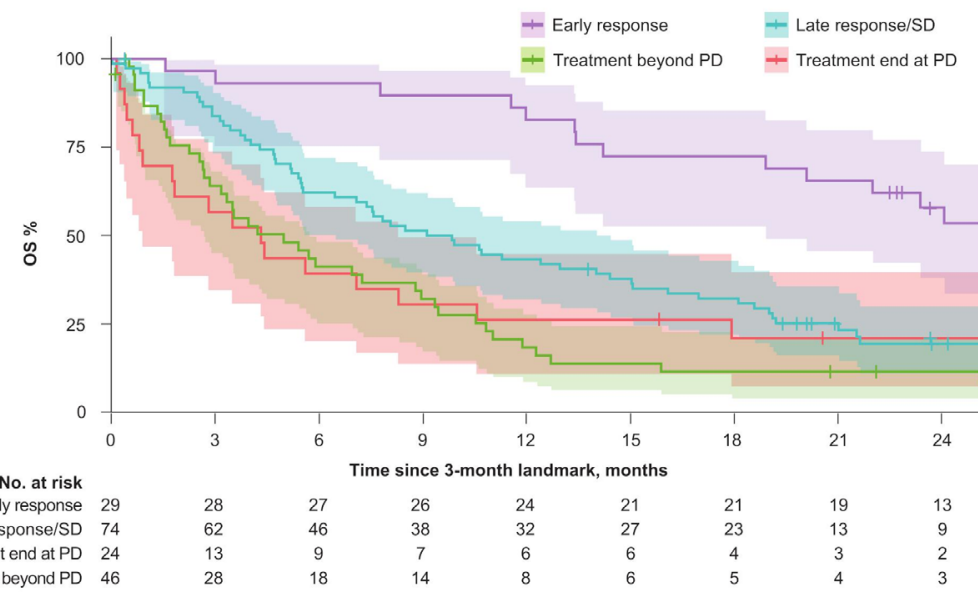

\begin{tabular}{|l|c|c|}
\hline \multicolumn{1}{|c|}{ Subgroup } & $\mathrm{N}$ & Median OS $(95 \% \mathrm{Cl})$, months \\
\hline Early responders & 29 & $\mathrm{NR}(18.9-\mathrm{NR})$ \\
Late response/SD & 74 & $9.5(6.5-14.4)$ \\
Treatment end at PD & 24 & $4.3(0.9-8.3)$ \\
Treatment beyond PD & 46 & $5.0(2.8-8.8)$ \\
\hline
\end{tabular}

Figure 3 Landmark analysis of overall survival beyond 3 months in postplatinum patients according to objective response (complete or partial response (CR/PR)) at 3 months. (A) Comparison of patients with (i) CR/PR by 3 months or (ii) no CR/PR by 3 months. (B) Comparison of patients with (i) CR/PR by 3 months, (ii) CR/PR after 3 months or stable disease (SD) by 3 months or (iii) best response of progressive disease (PD; before/after 3 months) or not evaluable. (C) Comparison of patients with (i) CR/ PR by 3 months, (ii) CR/PR after 3 months or SD by 3 months, (iii) best response of PD with end of treatment at PD or (iv) best response of PD with treatment beyond progression. NR, not reached. 
treatment would have also been based on patient characteristics at the time of PD. OS findings were confirmed by a sensitivity analysis that used an earlier landmark time point ( 7 weeks).

\section{Safety}

Safety findings were consistent with the 6-month analysis of this population $(\mathrm{N}=249) .{ }^{10}$ Treatment-related AEs (TRAEs) of any grade occurred in 177 patients (71.1\%), which included 11 additional patients compared with the 6-month analysis (online additional file 7). Grade $\geq 3$ TRAEs occurred in 29 patients $(11.6 \%$; 8 additional patients compared with the 6 -month analysis). The most common grade $\geq 3$ TRAE was fatigue (four $(1.6 \%)$ ); all other grade $\geq 3$ TRAEs occurred in one to two patients only. irAEs occurred in 51 patients (20.5\%; 17 additional patients compared with the 6-month analysis), including $12(4.8 \%)$ who had grade $\geq 3$ events (online additional file 8 ). The most commonly occurring categories of irAEs (any grade) were immune-related rash (28 (11.2\%), including various rash/pruritus AEs) and immunerelated thyroid disorders (13 (5.2\%), including hypothyroidism, hyperthyroidism and blood thyroid-stimulating hormone increased). IRRs and related events (based on an expanded definition) occurred in 78 patients $(31.3 \%)$, including grade $\geq 3$ events in three $(1.2 \%)$. Treatment-related death occurred in one patient $(0.4 \%)$, due to pneumonitis in a patient with ongoing, treatmentunrelated Clostridium difficile colitis and diverticulitis, as reported previously.
In an exploratory post hoc analysis, frequencies of the TRAEs examined were generally similar in high-risk patient subgroups compared with the overall population (table 2). In particular, patients with renal insufficiency or upper tract disease did not experience significant increases in serum creatinine, patients with liver metastases did not have an increased risk of hepatic events, and incidences of pneumonitis, gastrointestinal events (diarrhea and colitis), endocrine events (hypothyroidism and adrenal insufficiency) and IRRs were not elevated in any subgroup examined.

\section{DISCUSSION}

In this updated analysis with $\geq 2$ years of follow-up, avelumab showed durable antitumor activity in patients with previously treated advanced UC. The confirmed ORR in postplatinum patients was $16.5 \%$, including CR in $4.1 \%$. The median DOR was 20.5 months, and $65.4 \%$ of responses were maintained for $\geq 12$ months. Since this trial was initiated, the therapeutic landscape in advanced UC has changed following the regulatory approval of avelumab and four other anti-PD-L1/PD-1 agents in the USA and other countries. The efficacy of avelumab cannot be compared directly with other agents because of differences in patient populations, time frames of enrollment and study designs; however, ORRs appear to be consistent across approved anti-PD-L1/PD-1 agents in the postplatinum setting. ${ }^{781113}$ Although the ORR with avelumab was

Table 2 Treatment-related adverse events and infusion-related reactions in high-risk subgroups

\begin{tabular}{|c|c|c|c|c|c|c|}
\hline & \multirow[b]{2}{*}{$\begin{array}{l}\text { All patients } \\
(\mathrm{N}=249)\end{array}$} & \multicolumn{5}{|c|}{ High-risk subgroups } \\
\hline & & $\begin{array}{l}\text { Liver } \\
\text { metastases } \\
(n=84)\end{array}$ & $\begin{array}{l}\text { Renal } \\
\text { insufficiency } \\
\text { ( } n=113 \text { ) }\end{array}$ & $\begin{array}{l}\text { Upper tract } \\
\text { disease } \\
(n=58)\end{array}$ & $\begin{array}{l}\text { Elderly } \\
(n=73)\end{array}$ & $\begin{array}{l}\text { Low } \\
\text { albumin } \\
(n=46)\end{array}$ \\
\hline \multicolumn{7}{|l|}{ Renal events, n (\%) } \\
\hline Increased creatinine & $25(10.0)$ & $7(8.3)$ & $12(10.6)$ & $3(5.2)$ & $8(11.0)$ & $5(10.9)$ \\
\hline Renal/urinary disorders & $72(28.9)$ & $22(26.2)$ & $30(26.5)$ & $16(27.6)$ & $23(31.5)$ & $11(23.9)$ \\
\hline Pneumonitis, n (\%) & $9(3.6)$ & $2(2.4)^{\star}$ & $2(1.8)$ & $2(3.4)$ & $2(2.7)$ & $3(6.5)$ \\
\hline \multicolumn{7}{|l|}{ Gastrointestinal events, n (\%) } \\
\hline Diarrhea & $55(22.1)$ & $19(22.6)$ & $25(22.1)$ & $10(17.2)$ & $16(21.9)$ & $4(8.7)$ \\
\hline Colitis & $1(0.4)$ & 0 & 0 & 0 & $1(0.4)$ & 0 \\
\hline \multicolumn{7}{|l|}{ Hepatic events, n (\%) } \\
\hline Increased AST level & $18(7.2)$ & $11(13.1)$ & $9(8.0)$ & $2(3.4)$ & $5(6.8)$ & $3(6.5)$ \\
\hline Increased ALT level & $9(3.6)$ & $4(4.8)$ & $6(5.3)$ & $1(1.7)$ & $5(6.8)$ & $3(6.5)$ \\
\hline Autoimmune hepatitis & $1(0.4)$ & 0 & 0 & 0 & 0 & 0 \\
\hline \multicolumn{7}{|l|}{ Endocrine events, n (\%) } \\
\hline Hypothyroidism & $11(4.4)$ & $1(1.2)$ & $2(1.8)$ & $3(5.1)$ & $2(2.7)$ & $1(2.2)$ \\
\hline Adrenal insufficiency & $2(0.8)$ & $1(1.2)$ & $1(0.9)$ & $1(1.7)$ & 0 & 0 \\
\hline Infusion-related reaction, n (\%) & $60(24.1)$ & $23(27.4)$ & $23(20.4)$ & $15(25.9)$ & $21(28.8)$ & $13(28.3)$ \\
\hline
\end{tabular}

${ }^{*}$ One patient died due to pneumonitis.

ALT, alanine aminotransferase; AST, aspartate aminotransferase. 
numerically higher in patients with PD-L1+ versus PD-L1tumors $(23.8 \%$ vs $12.3 \%$, respectively), ORRs in both subgroups exceeded the historical, $\sim 10 \%$ ORR benchmark for postplatinum chemotherapy ${ }^{11} 29$ and median OS was similar between subgroups. In phase III trials of other antiPD-1/PD-L1 agents in the postplatinum setting, PD-L1 expression was not found to be a predictive biomarker. ${ }^{113}$ In first-line treatment of patients with cisplatin-ineligible UC, however, agents approved as monotherapy without prior chemotherapy (atezolizumab and pembrolizumab) are indicated only for patients with PD-L1+tumors.

In the exploratory post hoc analyses, avelumab showed antitumor activity in subgroups defined by characteristics associated with a poor prognosis or high-risk disease, suggesting that various known prognostic factors are not associated with response to avelumab. Compared with the overall population, ORRs with avelumab were numerically lower in patients with liver or visceral metastases or low albumin levels, but not in patients with low creatinine clearance levels or upper tract disease. Historically, patients with these characteristics have had poor outcomes with chemotherapy ${ }^{16}{ }^{30}$; thus, avelumab and other anti-PD-1/PD-L1 agents should be considered as treatment options for these patients. Unlike ORR findings, DCRs and PFS were similar in subgroups defined by albumin level. From a statistical perspective, trying to identify subgroups of patients with enhanced treatment effects introduces a high probability of finding false positives, particularly when there are no adjustments for multiple testing. To reduce the risk of false positives, subgroups should be defined prior to analysis, or a specific data-driven selection procedure should be predefined. ${ }^{27}$ For these analyses, a tree-based cut-off search with recursive partitioning was used. Despite using these established approaches, findings from the post hoc analyses should be interpreted with caution.

In other exploratory landmark analyses, there was no difference in response durability with avelumab in patients with response by week 7 compared with those who had a later response. However, treatment response (PR or CR) by 3 months appeared to be associated with longer OS than SD or later response (after 3 months), and OS in both subgroups was longer than in those who did not respond or achieve SD. Nevertheless, we also observed that some patients had long durations of stable disease with avelumab and some benefitted from continued treatment beyond initial RECIST-defined progression. Given the hypothesis-generating nature and limitations of the exploratory analyses, patients should remain on treatment until disease progression or unacceptable toxicity, per US FDA or local labeling.

With extended follow-up, avelumab continued to show an acceptable safety profile, including a low rate of grade $\geq 3$ TRAEs, and there were no new safety signals compared with an earlier analysis in this population or studies of avelumab in other tumor types. Additionally, occurrence of TRAEs in high-risk subgroups was consistent with the overall patient population.
Overall, these findings confirm the efficacy and safety seen in prior analyses of avelumab in patients with advanced UC and disease progression following platinum-based therapy. Initial results from this study led to a randomized phase III trial (JAVELIN Bladder 100; NCT02603432) to assess avelumab plus best supportive care as maintenance therapy versus best supportive care alone in patients with advanced UC without disease progression after 4-6 cycles of first-line platinum-based chemotherapy. It has been reported that the trial met its primary end point by showing significantly longer OS in the avelumab arm versus control arm (median 21.4 vs 14.3 months, respectively; HR 0.69 (95\% CI 0.56 to 0.86 ); $\mathrm{p}=0.0005){ }^{31}$ Together, these findings provide a further illustration of the clinical activity of avelumab in the treatment of advanced UC. Following the regulatory approval of avelumab as first-line maintenance and its inclusion in treatment guidelines, ${ }^{19} 3233$ it is likely that an increasing proportion of patients with advanced UC will receive avelumab in the first-line maintenance setting.

\section{Author affiliations}

${ }^{1}$ Genitourinary Malignancies Branch, Center for Cancer Research, National Cancer Institute, National Institutes of Health, Bethesda, Maryland, USA

${ }^{2}$ Nevada Cancer Research Foundation, Las Vegas, Nevada, USA

${ }^{3}$ Sarah Cannon Research Institute, Nashville, Tennessee, USA

${ }^{4}$ Associates in Oncology, Rockville, Maryland, USA

${ }^{5}$ HonorHealth Research Institute, Scottsdale, Arizona, USA

${ }^{6} \mathrm{Hematology} / 0 \mathrm{ncology}$, The University of Oklahoma Stephenson Cancer Center,

Oklahoma City, Oklahoma, USA

${ }^{7}$ Department of Medicine, Medical University of South Carolina, Charleston, South Carolina, USA

${ }^{8}$ Department of Medical Oncology, Sint-Augustinus Hospital Oncology Centre, Antwerp, Belgium

${ }^{9}$ Department of Internal Medicine, Seoul National University College of Medicine, Seongnam, Republic of Korea

${ }^{10} \mathrm{Hematology}$ Oncology, Oregon Health \& Science University, Portland, Oregon, USA

${ }^{11}$ Department of General Medical Oncology, University Hospitals Leuven, Leuven, Belgium

${ }^{12}$ Laboratory of Experimental Oncology, KU Leuven, Leuven Cancer Institute, Leuven, Belgium

${ }^{13}$ Henry Ford Cancer Institute, Detroit, Michigan, USA

${ }^{14}$ Department of Medical Oncology, Bordeaux University Hospital, Bordeaux, France

${ }^{15}$ EMD Serono Research \& Development Institute, Inc, Billerica, Massachusetts, USA; a business of Merck KGaA, Darmstadt, Germany

${ }^{16}$ EMD Serono, Inc, Rockland, Massachusetts, USA; a business of Merck KGaA, Darmstadt, Germany

${ }^{17}$ Florida Cancer Specialists/Sarah Cannon Research Institute, Sarasota, Florida, USA

Correction notice This article has been corrected since it was published online. The affiliations were re-ordered.

Twitter Andrea B Apolo @apolo_andrea and James L Gulley @gulleyj1

Acknowledgements The authors would like to thank the patients and their families, investigators, co-investigators and study teams at each of the participating centers and at Merck KGaA, Darmstadt, Germany, and EMD Serono, Inc.; a business of Merck KGaA, Darmstadt, Germany. Medical writing support was provided by ClinicalThinking and funded by Merck KGaA, Darmstadt, Germany, and Pfizer.

Contributors Conception and design: ABA, JM, GP, MR, JLG. Provision of study materials or patients: ABA, JAE, JRI, MA, MSG, RA, TG, LD, K-WL, MT, PS, DW, $A R, J L G, M R P$. Collection and assembly of data: all authors. Data analysis and interpretation: all authors. Manuscript writing: all authors. Final approval of manuscript: all authors. 
Funding This trial was sponsored by Merck KGaA, Darmstadt, Germany, as part of an alliance between Merck KGaA and Pfizer Inc. ABA and JLG received research funding from the National Cancer Institute's Center for Cancer Research.

Competing interests $A B A$ has no relationships to disclose. JAE has no relationships to disclose. JRI has received research funding from Aileron Therapeutics, ARMO BioSciences, AstraZeneca, BioMed Valley Discoveries, Bristol Myers Squibb, Calithera Biosciences, Celldex, eFFECTOR Therapeutics, Genentech/ Roche, GlaxoSmithKline, Immunocore, Janssen Oncology, Medlmmune, Merck and Co., Novartis, Pfizer, Phosplatin Therapeutics, Roche, and TESARO. MA has no relationships to disclose. MSG reports having stocks and other ownership interests in Caremission and WCCT Global; has received research funding from AbbVie, Acetylon, Aduro Biotech, Advaxis, Amgen, Array BioPharma, BeiGene, BioLineRx, Calithera Biosciences, CanBas, Celgene, Celldex, Corcept Therapeutics, CtyomX Therapeutics, Deciphera, Driver Group, Endocyte, ESSA, Five Prime Therapeutics, FujiFilm, Genentech/Roche, Gilead Sciences, GlaxoSmithKline, Halozyme, Hengrui Therapeutics, Inanovate, Incyte, Lilly, Lilly/ImClone, MabVax, Macrogenics, Medlmmune, Merck KGaA (Darmstadt, Germany), Merrimack, Millennum, Minneamrita Therapeutics, Nektar, Novartis, Novita Pharmaceuticals, OncoMed, Pfizer, Phoenix Biotech, Plexxikon, Proderm IQ, Samumed, Seattle Genetics, Sirtex Medical, Strategia Therapeutics, Syndax, SynDevRx, TESARO, Tokai Pharmaceuticals, Tolero Pharmaceuticals, Toray Industries, TRACON Pharma, Trovagene, and Verastem; and reports patents, royalties or other intellectual property for patient-on-patient selection for clinical trials. RA has no relationships to disclose. TG has received research funding from Ferring Pharmaceuticals. LD has no relationships to disclose. K-WL has received research funding from Array BioPharma, ASLAN Pharmaceuticals, AstraZeneca/Medlmmune, Five Prime Therapeutics, Green Cross Corp., LSK BioPharma, MacroGenics, Merck KGaA (Darmstadt, Germany), MSD, Ono Pharmaceutical, Pfizer, and Pharmacyclics. MHT reports serving as a consultant or advisor for ArQule, Array BioPharma, Bayer, Blueprint Medicines, Bristol Myers Squibb, Eisai, Loxo, and Novartis; and is a member of a speaker's bureau for Bristol Myers Squibb, and Eisai. PS reports serving as a consultant or advisor for Blueprint Medicines, Eisai, Ellipses Pharma, Epizyme, Ipsen, Lilly, Loxo, PIQUR Therapeutics, and Plexxikon; and has received research funding from Blueprint Medicines, Boehringer Ingelheim, CoBioRes, Eisai, Exelixis, G1 Therapeutics, Lilly, Novartis, PharmaMar, and Plexxikon. DW reports serving as a consultant or advisor for Merck and Co.; and has received reimbursement for travel, accommodation and expenses from Merck and Co. AR has received honoraria from Bristol Myers Squibb, Ipsen, Merck KGaA (Darmstadt, Germany), MSD, Novartis, and Pfizer; reports serving as a consultant or advisor for Bristol Myers Squibb, Ipsen, Novartis, Pfizer, and Roche; has received research funding from Novartis and Pfizer; and has received reimbursement for travel, accommodation and expenses from Bristol Myers Squibb, MSD, Novartis, and Pfizer. JM reports employment at EMD Serono Research \& Development Institute, Inc.; a business of Merck KGaA, Darmstadt, Germany. GP reports employment at EMD Serono, Inc.; a business of Merck KGaA, Darmstadt, Germany. MR reports employment at EMD Serono Research \& Development Institute, Inc.; a business of Merck KGaA, Darmstadt, Germany. JLG has received research funding from Astellas/Medivation, Bavarian Nordic, Bristol Myers Squibb, EMD Serono (a business of Merck KGaA, Darmstadt, Germany), Incyte, Janssen, Merck \& Co, Immunity Bio, and Pfizer. MRP is a member of a speaker's bureau for Celgene, Exelixis, Genentech/Roche, and Taiho Pharmaceutical.

\section{Patient consent for publication Not required.}

Ethics approval The trial was conducted in accordance with the ethics principles of the Declaration of Helsinki and the International Council for Harmonization Guidelines on Good Clinical Practice. The protocol was approved by the institutional review board or independent ethics committee of each center. All patients provided written informed consent before enrollment.

Provenance and peer review Not commissioned; externally peer reviewed.

Data availability statement Data are available on reasonable request. For all new products or new indications approved in both the European Union and the USA after January 1, 2014, Merck KGaA, Darmstadt, Germany, will share patient-level and study-level data after deidentification, as well as redacted study protocols and clinical study reports from clinical trials in patients. These data will be shared with qualified scientific and medical researchers, on researchers' request, as necessary for conducting legitimate research. Such requests must be submitted in writing to the company's data sharing portal. More information can be found at https:// www.merckgroup.com/en/research/our-approach-to-research-anddevelopment/ healthcare/clinical-trials/commitment-responsible-data-sharing.html. Where Merck $\mathrm{KGaA}$ has a coresearch, codevelopment or comarketing/copromotion agreement or where the product has been out-licensed, it is recognized that the responsibility for disclosure may be dependent on the agreement between parties. Under these circumstances, Merck KGaA will endeavor to gain agreement to share data in response to requests.

Open access This is an open access article distributed in accordance with the Creative Commons Attribution Non Commercial (CC BY-NC 4.0) license, which permits others to distribute, remix, adapt, build upon this work non-commercially, and license their derivative works on different terms, provided the original work is properly cited, appropriate credit is given, any changes made indicated, and the use is non-commercial. See http://creativecommons.org/licenses/by-nc/4.0/.

\section{ORCID iDs}

Andrea B Apolo http://orcid.org/0000-0001-9409-1836

Alain Ravaud http://orcid.org/0000-0001-9455-6744

James L Gulley http://orcid.org/0000-0002-6569-2912

\section{REFERENCES}

1 Bellmunt J, Choueiri TK, Fougeray R, et al. Prognostic factors in patients with advanced transitional cell carcinoma of the urothelial tract experiencing treatment failure with platinum-containing regimens. J Clin Oncol 2010;28:1850-5.

2 Akdogan B, Dogan HS, Eskicorapci SY, et al. Prognostic significance of bladder tumor history and tumor location in upper tract transitional cell carcinoma. J Urol 2006;176:48-52.

3 Cao J, Zhao X, Zhong Z, et al. Prognostic value of pre-operative renal insufficiency in urothelial carcinoma: a systematic review and meta-analysis. Sci Rep 2016;6:35214.

4 Salah S, Lee J-L, Rozzi A, et al. Second-line chemotherapy in older patients with metastatic urothelial carcinoma: pooled analysis of 10 second-line studies. Clin Genitourin Cancer 2017;15:e563-71.

5 Sonpavde G, Pond GR, Rosenberg JE, et al. Improved 5-factor prognostic classification of patients receiving salvage systemic therapy for advanced urothelial carcinoma. J Urol 2016;195:277-82.

6 Apolo AB, Ostrovnaya I, Halabi S, et al. Prognostic model for predicting survival of patients with metastatic urothelial cancer treated with cisplatin-based chemotherapy. J Natl Cancer Inst 2013;105:499-503.

7 Powles T, O'Donnell PH, Massard C, et al. Efficacy and safety of durvalumab in locally advanced or metastatic urothelial carcinoma: updated results from a phase 1/2 open-label study. JAMA Oncol 2017;3:e172411.

8 Sharma P, Retz M, Siefker-Radtke A, et al. Nivolumab in metastatic urothelial carcinoma after platinum therapy (CheckMate 275): a multicentre, single-arm, phase 2 trial. Lancet Oncol 2017;18:312-22.

9 Apolo AB, Infante JR, Balmanoukian A, et al. Avelumab, an antiprogrammed death-ligand 1 antibody, in patients with refractory metastatic urothelial carcinoma: results from a multicenter, phase $\mathrm{lb}$ study. J Clin Oncol 2017;35:2117-24.

10 Patel MR, Ellerton J, Infante JR, et al. Avelumab in metastatic urothelial carcinoma after platinum failure (JAVELIN Solid Tumor): pooled results from two expansion cohorts of an open-label, phase 1 trial. Lancet Oncol 2018;19:51-64.

11 Bellmunt $\mathrm{J}$, de Wit R, Vaughn DJ, et al. Pembrolizumab as secondline therapy for advanced urothelial carcinoma. $N$ Engl J Med 2017;376:1015-26.

12 Sharma P, Callahan MK, Bono P, et al. Nivolumab monotherapy in recurrent metastatic urothelial carcinoma (CheckMate 032): a multicentre, open-label, two-stage, multi-arm, phase 1/2 trial. Lancet Oncol 2016;17:1590-8.

13 Powles T, Durán I, van der Heijden MS, et al. Atezolizumab versus chemotherapy in patients with platinum-treated locally advanced or metastatic urothelial carcinoma (IMvigor211): a multicentre, openlabel, phase 3 randomised controlled trial. Lancet 2018;391:748-57.

14 Balar AV, Castellano D, O'Donnell PH, et al. First-line pembrolizumab in cisplatin-ineligible patients with locally advanced and unresectable or metastatic urothelial cancer (KEYNOTE-052): a multicentre, singlearm, phase 2 study. Lancet Oncol 2017;18:1483-92.

15 Keilholz U, Mehnert JM, Bauer S, et al. Avelumab in patients with previously treated metastatic melanoma: phase $1 \mathrm{~b}$ results from the JAVELIN Solid Tumor trial. J Immunother Cancer 2019;7:12.

16 Gulley JL, Rajan A, Spigel DR, et al. Avelumab for patients with previously treated metastatic or recurrent non-small-cell lung cancer (JAVELIN Solid Tumor): dose-expansion cohort of a multicentre, open-label, phase 1b trial. Lancet Oncol 2017;18:599-610.

17 Disis ML, Taylor MH, Kelly K, et al. Efficacy and safety of avelumab for patients with recurrent or refractory ovarian cancer: phase $1 \mathrm{~b}$ results from the JAVELIN Solid Tumor trial. JAMA Oncol 2019;5:393-401. 
18 Chung HC, Arkenau H-T, Lee J, et al. Avelumab (anti-PD-L1) as first-line switch-maintenance or second-line therapy in patients with advanced gastric or gastroesophageal junction cancer: phase $1 \mathrm{~b}$ results from the JAVELIN Solid Tumor trial. J Immunother Cancer 2019;7:30.

19 EMD Serono. Bavencio (avelumab) [prescibing information]. Rockland, MA: EMD Serono, a business of Merck KGaA, Darmstadt, Germany, 2020.

20 Mushti SL, Mulkey F, Sridhara R. Evaluation of overall response rate and progression-free survival as potential surrogate endpoints for overall survival in immunotherapy trials. Clin Cancer Res 2018;24:2268-75.

21 Ritchie G, Gasper H, Man J, et al. Defining the most appropriate primary end point in phase 2 trials of immune checkpoint inhibitors for advanced solid cancers: a systematic review and meta-analysis. JAMA Oncol 2018;4:522-8.

22 Abdel-Rahman O. Surrogate end points for overall survival in trials of PD-(L)1 inhibitors for urinary cancers: a systematic review. Immunotherapy 2018;10:139-48.

23 Roubaud G, Brouste V, Beuzeboc P, et al. Early objective response may not be a prognostic factor of survival for patients with metastatic urothelial carcinoma: from a retrospective analysis of a cohort of 113 patients. J Negat Results Biomed 2015;14:18.

24 Hassan R, Thomas A, Nemunaitis JJ, et al. Efficacy and safety of avelumab treatment in patients with advanced unresectable mesothelioma: phase $1 \mathrm{~b}$ results from the JAVELIN Solid Tumor trial. JAMA Oncol 2019;5:351-7.

25 D'Angelo SP, Hunger M, Brohl AS, et al. Early objective response to avelumab treatment is associated with improved overall survival in patients with metastatic Merkel cell carcinoma. Cancer Immunol Immunother 2019;68:609-18.
26 Heery CR, O'Sullivan-Coyne G, Madan RA, et al. Avelumab for metastatic or locally advanced previously treated solid tumours (JAVELIN Solid Tumor): a phase 1A, multicohort, dose-escalation trial. Lancet Oncol 2017;18:587-98.

27 Foster JC, Taylor JMG, Ruberg SJ. Subgroup identification from randomized clinical trial data. Stat Med 2011;30:2867-80.

28 Kazandjian D, Blumenthal G, Khozin S, et al. An exploratory responder analysis of best RECIST response and survival in patients with metastatic squamous NSCLC treated with nivolumab. J Thorac Oncol 2015;10:S234.

29 Bellmunt J, Théodore C, Demkov T, et al. Phase III trial of vinflunine plus best supportive care compared with best supportive care alone after a platinum-containing regimen in patients with advanced transitional cell carcinoma of the urothelial tract. J Clin Oncol 2009;27:4454-61.

30 von der Maase H, Sengelov L, Roberts JT, et al. Long-term survival results of a randomized trial comparing gemcitabine plus cisplatin, with methotrexate, vinblastine, doxorubicin, plus cisplatin in patients with bladder cancer. J Clin Oncol 2005;23:4602-8.

31 Powles T, Park SH, Voog E, et al. Avelumab maintenance therapy for advanced or metastatic urothelial carcinoma. N Engl J Med 2020;383:1218-30.

32 National Comprehensive Cancer Network. NCCN clinical practice guidelines in oncology: bladder cancer, 2020. Available: https://www. nccn.org/professionals/physician_gls/pdf/bladder.pdf [Accessed 21 Jul 2020].

33 Bellmunt J, Leow JJ, Wiegel T, et al. eUpdate - bladder cancer: ESMO clinical practice guidelines for diagnosis, treatment and follow-up. Available: https://www.esmo.org/guidelines/genitourinarycancers/bladder-cancer/eupdate-bladder-cancer-treatmentrecommendations4 [Accessed 21 Jul 2020]. 